\title{
Realizing the value proposition of hosted DAM solutions - A case study
}

\begin{abstract}
Mukul Krishna
is widely recognized as an expert and thought leader in the Digital Media industry with his primary expertise being in the Digital Asset Management, Video Server, Streaming and Encoding markets. He is responsible for the digital media practice at Frost and Sullivan. He has extensively written on, analyzed and managed projects within the content management, rights management, video server, streaming, encoding, digital video recorder, storage and interactive kiosk markets. Apart from analyzing these markets, he has also lead consulting projects in these fields for key Frost \& Sullivan clients. He is a regular speaker and panelist at industry conferences and gives quarterly webinars at Frost and Sullivan about the state of the digital media industry. $\mathrm{He}$ also regularly participates in brainstorming strategy sessions with Frost and Sullivan clients.
\end{abstract}

Keywords: hosted DAM, DAM software as a service (SaaS), return on investment, value proposition, widen, Reebok

Abstract The value proposition of DAM and more importantly DAM as software as a service (SaaS) is no longer a "leap of faith." DAM is now a "must-have" for organizations looking at gaining marketing efficiency. Market evolution aided by infrastructural and technology advancements in storage, security, bandwidth, rich internet applications and web services have created a landscape that puts customers in a situation that they never were in before - they have choices. Customers have a wide array of solutions and many flavors of DAM to choose from - both, installed and hosted. With a proactive vendor community that is constantly evolving with the demands of its customers, this market is positioned as never before to see robust growth in the years to come. Journal of Digital Asset Management (2007) 3, 50-54. doi:10.1057/palgrave.dam.3650059

Mukul Krishna Digital Media Practice, Frost \& Sullivan, 7550 lh-10 West, Suite 400, San Antonio, TX 78229 USA

Tel: +12102473850 Fax: + 12103481003 Email: mkrishna@frost. com;

Web: http://www.frost.com

\section{SO WHAT IS WITH THE DAM HOSTED BUZZ?}

For too long installed DAM solutions have been perceived as technology tools for the elite corporation. Owing to this perception, DAM as a SaaS (software as a service) model has been seen as a cheap alternative for the SMB market to get its foot in the door and experience a few of the benefits that richer and larger entities have been experiencing through installed deployments. This could not be farther from the truth. In reality, each organization has it own unique set of requirements and it is only through a thorough needs assessment process that one can ascertain what would be the best path for an individual company (big or small) to take - installed or hosted and then chose a vendor accordingly.

The misconception of DAM as SaaS being the less wealthy company's only choice saw its genesis early in the market's inception when old school business philosophy dictated full control of all technology and business process tools and outsourcing was considered sacrilegious. This was further fueled by the fact that initially vendors offering such solutions were largely very small start-ups looking at creating a market presence. Deploying with the SMB market for these vendors was an easier proposition because the cost savings through a SaaS model immediately struck a chord with the smaller, price-sensitive company and coupled with shorter sales cycles the SMB market was an ideal target.

The market has evolved tremendously since then. Some of the biggest challenges to the SaaS model have traditionally been around security, robustness and infrastructure. Technological advances, declining storage costs and the proliferation of high-speed broadband have transformed the business landscape and the $\mathrm{SaaS}$ model is no longer seen as a risky option. Companies like Salesforce.com have paved the way for vendors offering various hosted applications for the enterprise. 
Based on analysis of the DAM market by Frost \& Sullivan, the emerging SaaS segment represented $\$ 35 \mathrm{~m}$ of the $\$ 350 \mathrm{~m}$ core solutions market in 2006, representing 10 per cent of the overall market. The DAM SaaS market is expected to expand at a very aggressive $30+$ per cent annual rate as opposed to the $20+$ per cent for installed solutions. The SaaS model has started gaining much wider acceptance across verticals and the robust growth has attracted many vendors to start exploring this model seriously. To put this growth is perspective, we need to look at market evolution and the value proposition of the DAM space.

\section{Market evolution}

DAM can be defined as a value chain of applications with loggers at one end, core DAM applications in the middle and digital delivery software and services at the other end. DAM is used across verticals in the management of digital content, which includes various file types including audio, video, graphics, text and more. DAM solutions enable the ingest, archiving/indexing, search retrieval, browsing, version control, annotation, repurposing, collaboration, display and transport of digital media.

The value proposition of deploying DAM is multifold. An end-to-end DAM solution can be seen as a value chain of applications namely: Logging/Archiving, Asset Management, Delivery and Online Content Management. The line that divides these applications is blurring rapidly. Just a few years back vendors had traditionally provided only one part of the DAM value chain to the customer (Figure 1), for example: loggers (Virage, Convera), DAM software (Widen,

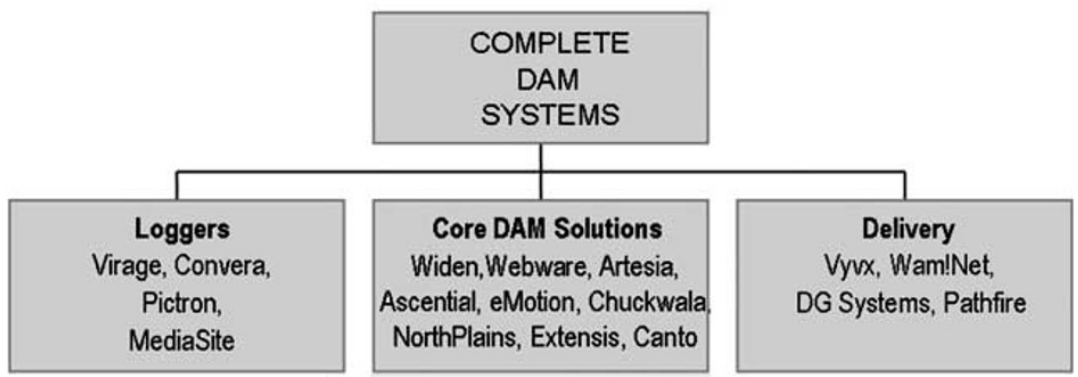

Figure 1: DAM value chain - before 2002

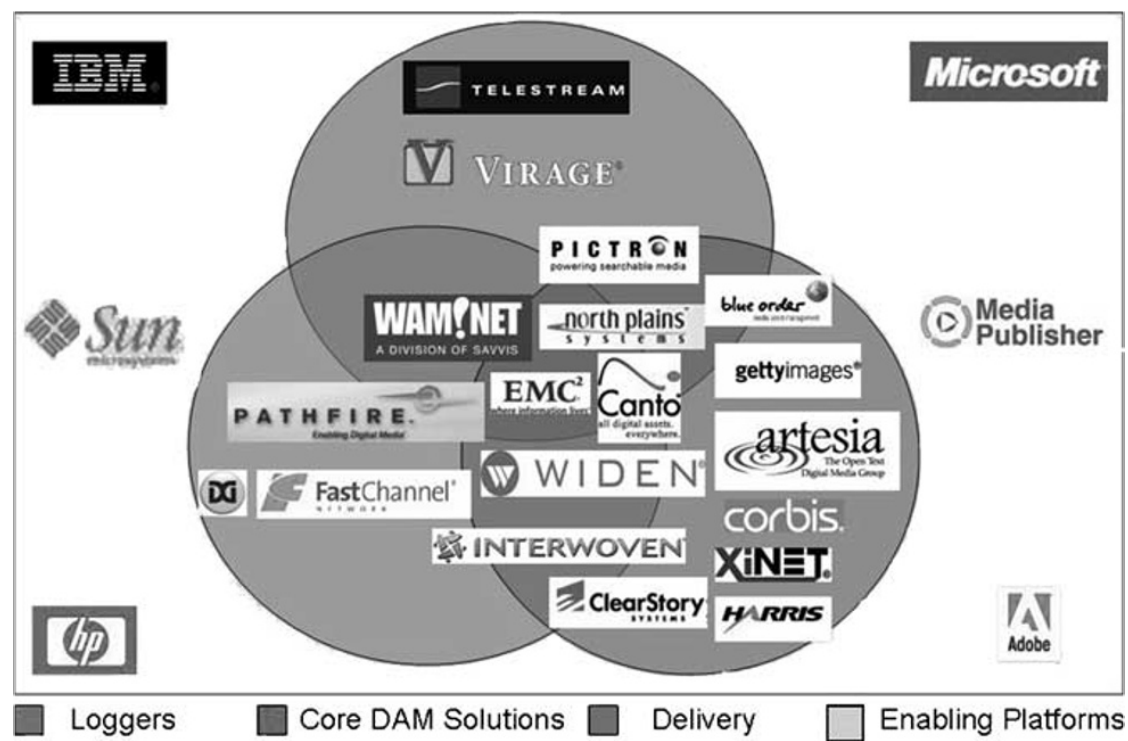

Figure 2: DAM value chain -2006 
ClearStory, NorthPlains, Artesia), Delivery

(WAM!NET, Vyvx).

Increasingly vendors are providing more functionality, bringing different parts of the DAM value chain together through their solutions (Figure 2), diminishing the line between loggers and core DAM solutions. The market is extremely fragmented and during the downturn most vendors had limited resources for growth. This has lead to consolidation as vendors are looking to increase their target market and gain access to more resources for growth.

Consolidation has been mainly with companies in allied markets such as WCM to form the Enterprise Content Management (ECM) space and with technology vendors providing solutions for the Media \& Entertainment space.

\section{The value proposition - Show me the money!}

Once assets are digitized and have metadata associated to them they can be archived (logged) within a digital archive. This process itself yields tremendous cost savings as the organization ends up saving huge amounts of resources in moving from a physical archive to a digital repository. With constantly declining digital storage costs companies now do not have to deal with the heavy cost of real estate to sustain voluminous physical libraries nor on the associated cumbersome and resource-intensive maintenance requirements.

Archived digital assets are easily searchable through the associated metadata thus eliminating the cost of lost or misplaced work. Apart from helping with corporate compliance, this is also a great time saver when it comes to production time and in turn reduces overhead costs significantly while freeing up resources for increased productivity that would have traditionally been used to search assets.

Increasingly enterprises have moved towards centralization of their assets as they find it easier to manage digital assets and track them from a single location. This has created a great demand for efficient processes to manage these assets. Corporate entities across verticals have started seeing immense value in DAM as they have huge amounts of their branded intellectual property that need to be digitized, catalogued, managed, tracked and repurposed.

Beyond logging and at the core is the DAM process. The cost of lost or misplaced work has become a major cause of concern for corporations as the advantage of being able to reuse and repurpose existing searchable assets has become clear. Management of assets helps in control, governance and monetization through integration with the marketing and production workflows. Version control and authorization protocols add another layer of protection to the branded intellectual property - the digital assets. A DAM system now adds significantly to the bottom line by streamlining many marketing and corporate communication processes and thus reducing operation and production costs while ensuring a greater speed-to-market with uniform and controlled messaging.

Delivery of digital assets is fast becoming a part of the core asset management solutions as well. With increased regional centralization of large functional workgroups in media production, the need for a high degree of collaboration to get the finished product has become paramount. Organizations typically have started realizing efficiencies by centralizing the preparation of content at one facility, however other production work, such as graphic art, maybe done at another facility. As companies add more rich digital media to their brand strategy, file sizes are exploding. Stakeholders to these assets are many times geographically dispersed but still need to easily access and collaborate on the same assets from different locations. Through integration with third-party systems using web services and providing conditional access and version control, DAM systems have now become an ideal platform to enable such collaboration in a seamless workflow.

Using the hosted model for DAM, customers not only get the above-mentioned benefits and in some cases faster than through an installed deployment, but are also able to free-up valuable internal IT resources to do other productive tasks. Extensive Service Level Agreements and negotiated contracts also ensure continued stability of the business process with guaranteed uptime and the ability to bring the solution in-house if needed. 


\section{THE DAM SaaS VENDOR LAND- SCAPE}

Although DAM as SaaS has been around for a while, it is only now that we have started seeing a lot of customer traction as discussed earlier. Not only do we have specialized SaaS vendors for DAM such as Widen, Getty and Corbis among others, many of the traditional installed software vendors have also started getting their SaaS strategy in place now looking at the tremendous growth potential this space offers.

Widen (www.widen.com) has been a fixture in the graphic arts business since 1948 and took the leap towards providing DAM through a hosted model in 1998. Since then the company has been able to provide its customers - big and small - with tangible benefits through a highly evolved hosted DAM service. The value proposition offered by companies like Widen has been very real because of which companies like Reebok (www.reebok.com) are now fully committed to the SaaS model to have their DAM needs fulfilled.

Widen's product direction is geared up to serve three distinct functions; each supporting brand control while embracing rapid go-tomarket strategies. These three functions include:

- marketing execution;

- creative support;

- sales enablement.

Widen is positioning itself as a must-have technology within these spaces and the aggressive three-time-per-year release cycle creates an environment where customers are never again left hanging with a technology investment that gets antiquated rapidly. This proactive approach towards product upgrades and client support is fast characterizing the DAM space and has greatly helped the industry in coming out of the downturn and growing stronger each year.

Widen attributes their continued successes to listening to the unarticulated demand of the marketplace. Customers are trying to ask for it but they do not know exactly how to say it, which makes customer interaction and longterm investments in products and services critical to future successes.
Widen marketing and communications teams lead customer interaction and shape user requests to systems engineers. Widen systems are designed by internal software architects and development teams hosted with a sophisticated infrastructure. These internal elements make it easy for Widen to provide applications that constantly meet marketing, creative and sales objectives.

\section{REEBOK AND HOSTED DAM}

Reebok is a very marketing intensive company and being an apparel printing company, it has a huge amount of its brand equity in graphics and images. Most of the assets that Reebok deals with are Digital Samples - digital pictures of product that get distributed to Retailers and Teams for use in catalogues and on websites, as well as final graphic, design and techpack documents which are Adobe files that are the base description of Reebok's products. These files are very critical as they form the base of Reebok's product development process and product supply-chain process.

Reebok had been facing many challenges before Doug Rammel, Vice-President of Information Systems, Technology and Integration Operations for On-Field Apparel Group/Group Athletica, LLC looked at DAM. Reebok used a variety of shared drive and file transfer protocol technologies. None of which met the company's needs. The main pain-points for the company were centered on the confusion between versions because of the existence and creation of multiple copies of files and multiple generations of development files. This was compounded by lost or misplaced files because of problems in organization and duplication of work due to storage, retrieval and tracking of both jobs and files.

Rammel was quick to understand the nature of Reebok's challenges and looked at the DAM market for solutions. The company was fairly sophisticated in its selection and implementation process. "We did a fairly complete RFI process as part of its vendor selection which ended with a pilot program between the top two short listed vendors around Super Bowl XXV,' communicates Rammel.

Reebok finally chose Widen to address the company's DAM needs. Rammel states that 
Reebok selected Widen "because of experience with graphic files and the prepress industry and their willingness to customize the product and build it to fit Reebok's operations as they evolved." Through this implementation Reebok was able to streamline many of its processes and make them more cost-effective. The implementation brought in version control and eliminated the confusion around versions of files and designs. With a searchable digital archive, the need to procure physical samples prior to the production season for catalogue and website shoots has been eliminated thus reducing the time-to-market. By the creation of a digital library, which is able to tie into Quark and InDesign, Reebok has been able to leverage design files into current, up-to-date sales collateral assets. Some of the other tangible benefits the company has received include:

- Since inception, digital asset requests have increased an average of 89.81 per cent each year nearing 200,000 requests in 2006 alone.

- Requests for physical samples has dropped an average of 5.60 per cent each year equating to significant cost savings.

- Fulfillment of digital assets for field sales use is immediate, accommodating a specific selling situation to increase closing percentages as opposed to physical product sample use which takes anywhere from 5 to 60 days to get it in the hands of the salesperson.

- Over the last two years, each digital asset created has been repurposed over six times in its lifecycle versus a one-time use prior to Widen.
- Reduced the cost to Reebok's customers by 93 per cent for creation and distribution of brand images as a result of DAM.

- Reduced time from weeks to minutes for customers to get Reebok products to market through the use of Widen's hosted DAM to distribute market-ready images.

- Eliminated 44 per cent of costs related to distribution of digital assets by using Widen.

\section{THE BOTTOM LINE}

As seen in the above case study, the numbers Reebok has started seeing through this initiative are pretty significant and scream "Rapid ROI". The beauty of DAM is that it not only helps in significant cost reduction but now we have started getting tangible evidence of DAM going further to help in added revenue generation. The value proposition of DAM and more importantly DAM as SaaS is no longer a "leap of faith." DAM is now a "must-have" for organizations looking at gaining marketing efficiency.

Market evolution aided by infrastructural and technology advancements in storage, security, bandwidth, rich internet applications and web services have created a landscape that puts customers in a situation that they never were in before - they have choices. Customers have a wide array of solutions and many flavors of DAM to choose from - both, installed and hosted. With a proactive vendor community that is constantly evolving with the demands of its customers, this market is positioned as never before to see robust growth in the years to come. 